Rabaska

Revue d'ethnologie de l'Amérique française

\title{
Table ronde « Le patrimoine est-il sexiste ? »
}

\section{Véronique Stahn}

Volume 16, 2018

URI : https://id.erudit.org/iderudit/1051337ar

DOI : https://doi.org/10.7202/1051337ar

Aller au sommaire du numéro

Éditeur(s)

Société québécoise d'ethnologie

ISSN

1703-7433 (imprimé)

1916-7350 (numérique)

Découvrir la revue

Citer ce document

Stahn, V. (2018). Table ronde « Le patrimoine est-il sexiste ? ». Rabaska, 16,

218-221. https://doi.org/10.7202/1051337ar d'utilisation que vous pouvez consulter en ligne.

https://apropos.erudit.org/fr/usagers/politique-dutilisation/ 


\title{
Table ronde « Le patrimoine est-il sexiste ? »
}

\author{
VÉRONIQUe STAHN \\ Université du Québec à Montréal
}

La table ronde « Le patrimoine est-il sexiste » a réuni, le 15 mars 2018 au Centre de diffusion et d'expérimentation CDEX à l'Université du Québec à Montréal (UQÀM), plusieurs intervenants et intervenantes des milieux universitaire et professionnel autour d'une question qui fait de plus en plus l'actualité. Cet événement a été organisé conjointement par l'Institut de recherches et d'études féministes (IréF), l'Institut du patrimoine de l'UQÀM, le Centre de recherche Cultures-Arts-Sociétés (CELAT) et l'Institut du patrimoine culturel de l'Université Laval (IPAC).

La table ronde, présidée par Sylvie Cameron (candidate au doctorat en muséologie, UQÀM), était composée de Carole Charbonneau (muséologie autochtone), Valérie Perron (candidate à la maîtrise en arts visuels et médiatiques, UQÀM), Monique Provost (postdoctorante, Institut du patrimoine), Noémie Larue-Lapierre (Espace pour la vie), Michèle Rivet (ex-juge du tribunal des droits de la personne, Université de Sherbrooke), Marie Fraser (professeure, histoire de l'art, UQȦM) et Nada Guzin-Lukic (professeure, muséologie, Université du Québec en Outaouais/UQO). Elle a permis aux différentes intervenantes de répondre à trois questions en lien avec le sujet, suivi par une conversation libre avec l'auditoire.

L'idée de la table ronde, précisée en ouverture par Yves Bergeron, directeur de l'Institut du patrimoine, est venue à la fin de l'automne 2017, lorsque la porte-parole Manon Massé a annoncé que Québec Solidaire souhaitait faire disparaître le mot patrimoine de son programme. L'annonce par la députée, en novembre 2017, d'un débat sur le terme patrimoine, jugé trop masculin, lors du congrès de son parti, depuis abandonnée, avait alors causé une forte polémique publique. L'Institut du patrimoine s'était alors posé la question de savoir si un institut universitaire pouvait prendre position dans ce débat. Yves Bergeron et Habib Saidi, directeur de l'Institut du patrimoine culturel de l'Université Laval, ont décidé de questionner cette tendance, qui apparaît également en dehors des frontières du Québec, notamment en France, où la 
notion de patrimoine était également remise en question à l'occasion, entre autres, des Journées du patrimoine 2017. Cette table ronde a permis d'ouvrir le débat. Les intervenantes réunies autour de la table ont exposé leurs différents points de vue, en lien avec leurs expériences, leurs pratiques et leurs domaines d'intervention ou de connaissance.

La première à intervenir, Monique Provost, posa un point de vue d'ethnologue sur le patrimoine, en prenant en compte particulièrement le patrimoine immatériel. Pour elle, le patrimoine touche à une problématique linguistique et sémiologique, car le terme apporte une idée dans un groupe social donné : comme la société évolue, les mots utilisés évoluent également. Selon leur expérience, le sémiologue, l'historien ou le politicien portent un regard différent sur la notion. Pour Monique Provost, le patrimoine n'est pas sexiste par son origine sémiologique, mais par sa fonction pragmatique dans la société. Il faut s'interroger sur l'utilité de cette notion, car elle ne réfère pas à un groupe mais à une fonction socialement définie. M. Provost s'est opposée à la logique de substitut proposée par Manon Massé, car c'est une imposition politique alors qu'on doit assister à une évolution historique.

Carole Charbonneau a répondu, quant à elle, à la question à travers le patrimoine autochtone, un héritage du passé dont la tradition a été interrompue et qu'on doit retrouver pour l'avenir. Cet héritage passe par la langue : comment, par exemple, transmettre des contes et légendes sans la langue? Concernant la question sur le sexisme du patrimoine, elle estime qu'il est délicat d'en parler actuellement du point de vue autochtone, car ceux-ci sont en train de reconquérir leur patrimoine, qui collabore à leur guérison. Le patrimoine n'est, dans les sociétés autochtones, pas sexiste mais sexué, les mots « patrimoine » et « sexiste » n'existant pas dans leurs langues.

Valérie Perron, qui intervenait ensuite, possède une pratique féministe et de performance. Pour elle, les pratiques féministes ont beaucoup utilisé la performance en art. Les reenactments, par exemple, font leur entrée dans les musées et ont acquis une reconnaissance. Elle s'est interrogée sur le fait que, en tant que femme, on souhaite que notre héritage soit reconnu au-delà des mots patrimoine ou héritage. Le terme patrimoine a, pour elle, une connotation autoritaire et exclusive qui a fait que l'histoire des femmes a été longtemps écartée des institutions. La notion de patrimoine est une polyphonie qui n'est pas à traiter de façon linéaire : il faut repenser la façon de faire l'histoire, non linéaire, ce qui fera de facto évoluer le terme.

Noémie Larue-Lapierre est intervenue plutôt à titre personnel, comme témoin dans le domaine du travail. Pour elle, on retrouve une forte représentation de femmes dans la culture et la question est de savoir si le terme est sexiste alors que les travailleurs du patrimoine sont des femmes. Était-il sexiste par le passé ? Sûrement. On assiste sans doute à une évolution. 
Le malaise actuel autour du mot patrimoine, mot exclusif et basé sur l'héritage du père, a été mis en avant par Nada Guzin-Lukic. Ce malaise interpelait déjà l'UQO lors de la création du programme en muséologie il y a 10 ans : le titre «muséologie et patrimoines » a alors été proposé. Pour la professeure, il faut se demander de quelle manière la féminité interroge et interagit avec le patrimoine, tout en prenant en compte la notion de manière élargie, par exemple le patrimoine de l'humanité (UNESCO). Les enjeux du patrimoine sont complexes, car ce sont des notions politiques et exclusives, notamment lorsqu'elles touchent à l'identité. Une approche inclusive du patrimoine est à discuter en pratique et à aborder au niveau de l'hybridation des termes, car l'usage du mot est actuellement sexiste.

Pour Marie Fraser, la question posée est limitée, car elle appelle une réponse simple : oui ou non, et manque de documentation. Dans les faits, la constitution du patrimoine est sexiste : son analyse de la présence de femmes en art moderne et contemporain (à la biennale de Venise et dans les collections muséales), pour plusieurs pays, ainsi que la dénonciation du sexisme à travers l'art féministe, révèle la quasi-absence d'artistes féminines dans les milieux institutionnels de l'art contemporain, même si cette représentation augmente au fur et à mesure, bien que lentement.

Michèle Rivet a alimenté le débat du point de vue du droit. Elle a insisté, en introduction, sur l'importance d'un bâtiment nommé Judith-Jasmin à l'UQÀm et sur la coïncidence d'y tenir la table ronde, puis elle a présenté l'utilisation des mots en droit, domaine qui structure la société. Elle estime que, si l'on veut changer le terme patrimoine, cela doit passer par le droit, ce qui a été le cas pour plusieurs notions, par exemple pour la considération des femmes en tant que personnes ou pour les droits de la personne au Canada (plutôt que les droits de l'Homme). Dans tous les cas, le législateur donne des pistes ou cristallise ce que souhaite la société. Pour conclure, Michèle Rivet a souligné que le terme patrimoine est d'usage commun au Québec, mais n'a pas de connotation sexuée en droit : au niveau de la notion de propriété, le législateur a une vision économique de la personne ("Toute personne est titulaire d'un patrimoine », selon le Code civil), et il ne s'interroge pas sur le terme au niveau culturel.

La seconde partie de la table ronde ouvrait la discussion au public, qui a repris le débat autour de plusieurs questions initiées dans la première partie. Plusieurs idées se sont détachées des discussions, notamment sur la définition et l'utilisation du terme patrimoine, mais aussi sur les limites de tels débats et la justification de l'imposition d'un nouveau mot ou d'une autre utilisation par une personne politique.

Les échanges ont montré que le problème ne provient pas tant du mot 
patrimoine en lui-même que de ce qu'il symbolise dans notre société. Même si certaines participantes souhaitent son remplacement, c'est plutôt le système en entier qui est sexiste et porteur de discrimination, car la structure de décision patrimoniale est masculine et exclusive.

Maurizio Ruiz, doctorant en sémiologie à l'UQÀM, a interrogé le fait que, si le patrimoine est une chose qu'on assume collectivement, avec des valeurs qu'on partage, est-ce que la communauté serait prête à soutenir le fait que ces valeurs soient remises en question, notamment sur la base de la problématique sexiste ? Pour Marie Fraser, Manon Massé a posé un geste audacieux qui a eu le mérite de lancer le débat, ce qui indique que celui-ci est sorti du monde des spécialistes. Pour elle, l'usage du mot va évoluer avec la société, cependant un geste politique fort est le bienvenu pour faire avancer la question. Les institutions culturelles sont, selon Marie Fraser, de plus en plus tournées vers l'avenir et cherchent des stratégies pour mieux s'ancrer dans la société et constituer l'héritage culturel du futur autrement; il est cependant important de se remémorer le passé colonial et sexiste lié au terme patrimoine. Nadia Guzin-Lukic a ajouté qu'il est nécessaire de réactualiser le mot, mais également de penser à la préservation du patrimoine lié aux femmes, notamment en Europe de l'Est. Michèle Rivet a insisté, pour sa part, au besoin de changer les structures de gouvernance afin qu'elles deviennent plus inclusives.

Yves Bergeron a clôturé la table ronde en notant la complémentarité des interventions. Il a conclu en soulignant que les universitaires sont particulièrement concernés par deux types de patrimoine. Le premier a une relation au pouvoir et encadre la vie collective et culturelle de l'université ; il existe également sous forme d'industrie avec des enjeux économiques, ce qui a en retour des incidences sur l'université. Le second est le patrimoine comme objet d'études. Il concerne toutes les disciplines et dépasse le cadre légal. La responsabilité des universitaires est alors de pousser la recherche, que le patrimoine soit ou non intégré à la loi.

En conclusion, pour Yves Bergeron, la loi sur les « biens culturels » est plus pertinente que sur le « patrimoine culturel». Depuis le rapport Arpin (2000), on observe un retour du terme patrimoine, car il existe un consensus social sur cette notion, qui est beaucoup plus large que quelques monuments (notamment avec l'existence du patrimoine matériel et immatériel). La notion de patrimoine est, selon Yves Bergeron, redéfinie tous les vingt ans environ et n'est pas un terme encadré et définitif. Au Québec, le rapport amour/ haine avec ce terme est également cyclique. Finalement, le plus important, en accord avec la définition de l'Icom sur le patrimoine immatériel, est la culture collective. 